\title{
LDL Isolated from Plasma-Loaded Red Wine Procyanidins Resist Lipid Oxidation and Tocopherol Depletion
}

\author{
Cátia F. Lourenço, ${ }^{\dagger}$ Bruno Gago, ${ }^{\dagger}$ Rui M. Barbosa,$^{\dagger}$ Víctor de Freitas, ${ }^{\ddagger}$ \\ AND JOÃO LARANJINHA ${ }^{*}, \dagger$ \\ Faculty of Pharmacy and Center for Neurosciences and Cell Biology, University of Coimbra, \\ Coimbra, Portugal, and Chemistry Department, Faculty of Science, University of Porto, \\ Porto, Portugal
}

Dietary phenolic compounds may act as antioxidants in vitro, but because of structural modifications during absorption, its role based on concentrations high enough to afford an antioxidant protection needs to be re-evaluated. We have explored the hypothesis that red wine procyanidins interact with low density lipoproteins (LDL) and that, at this location, the phenolic compounds efficiently protect LDL from oxidation and maintain LDL $\alpha$-tocopherol at a high steady state concentration by recycling it back from the $\alpha$-tocopheroxyl radical. To this end, human plasma was supplemented with wine procyanidins and isolated LDL were challenged with a constant flux of peroxyl radicals. As compared with LDL from plasma-free procyanidins, those LDL better resisted lipid oxidation and exhibited longer lag-phases of $\alpha$-tocopherol consumption. The procyanidins, depending on their structure, were able to reduce the UV-induced $\alpha$-tocopherol radical in a micellar system, as evidenced by electron paramagnetic ressonance. Mechanistically, the protection of LDL was interpreted in terms of quenching of peroxyl radicals and the recycling of $\alpha$-tocopherol by the procyanidins bound to the lipoproteins. These results support the notion that, in human plasma, the procyanidins, via binding to LDL, may act as efficient local antioxidants.

KEYWORDS: Wine; procyanidins; LDL oxidation; $\alpha$-tocopherol recycling

\section{INTRODUCTION}

Evidences from epidemiology and biochemistry suggest potential health benefits of the consumption of phenolic compounds, including those from red wine, particularly in connection with diseases associated with oxidative events, most notably the oxidation of low density lipoproteins (LDL) in atherosclerosis $(1,2)$. LDL oxidative modification in the vascular wall seems to be a key factor in the pathogenesis of atherosclerosis, which is one of the most prevalent cause of morbidity and mortality in Western countries $(1,3)$.

Mechanistically, the health-promoting properties have been assigned to the well-known antioxidant activities of phenolic compounds, largely supported by their ability to scavenge free radicals and chelate redox-active metals, catalyzers of oxidative reactions (4). This rationale, however, has faced difficulties because phenolic compounds are extensively metabolized during absorption and reach the plasma in very low concentrations (5-7).

Alternative, pathways for the biological impact of phenolic compounds are being unraveled and include, among others, the

* To whom correspondence should be addressed. E-mail: laranjin@ci.uc.pt.

$\dagger$ University of Coimbra.

* University of Porto. interference with cell signaling pathways, with nitric oxide metabolism, and the modulation of enzyme activity (8-12).

From the biochemical antioxidant viewpoint, however, new concepts have also emerged, including the activity of the phenolic compounds at lipid-water interfaces, encompassing redox cycles with $\alpha$-tocopherol $(\alpha$-TOH) in membranes and lipoproteins and also with ascorbate in the water phase (13). Such a mechanism, exporting the radical character from the lipid to the water phase and maintaining the LDL $\alpha-\mathrm{TOH}$ at high steady state concentration, has been shown to afford a high antioxidant protection to LDL (14). Thus, considering its physicochemical properties, phenolic compounds may interact with membranes and lipoproteins peaking locally at high concentrations and exerting local antioxidant activity, as opposed to the low concentrations achieved when considering their isotropic dilution in blood plasma (for reviews see 15 and 16). From the physiological viewpoint, these properties of phenolic compounds would be particularly useful in the intima space where LDL is supposed to be sequestered from the plasmatic antioxidants (17).

Red wine contains a wide variety of phenolic substances, most of which are derived from the solid components of the grape berry, skin, and seeds and could be categorized as flavonoids and nonflavonoids. Among the flavonoids (e.g., flavonols, 
flavanols, and anthocyannins), procyanidins (flavan-3-ols) were identified as one of the most active phenolic groups in red wine $(18,10)$. Procyanidins are oligomers or polymers of monomeric flavan-3-ol units $((+)$-catechin and/or $(-)$-epicatechin) linked by an interflavan linkage established between the $\mathrm{C} 4$ carbon of the upper unit and the $\mathrm{C} 6$ or $\mathrm{C} 8$ carbon of the lower unit. Procyanidins and the remaining red wine phenolic compounds have been proposed to inhibit proatherogenic pathways, as they are known to increase resistance of LDL to peroxidation (19-22), to act as platelet aggregation modulator (23-25) and to promote nitric oxide-mediated vasorelaxation $(26,27)$. Whereas the exact mechanisms by which red wine polyphenols exert significant effects at the low concentrations achieved in vivo are not fully elucidated, it has been shown that red wine polyphenols interact with low density lipoproteins (LDL), protecting the particles from oxidation (28). Thus, we have established a model consisting of human plasma incubated with wine procyanidins and the evaluation of the oxidizability of isolated LDL in terms of lipid oxidation and $\alpha-\mathrm{TOH}$ recycling in order to mechanistically determine a local antioxidant activity for phenolic antioxidants at the LDL surface with potential physiological significance.

\section{MATERIALS AND METHODS}

Procyanidins Synthesis and Isolation. The procyanidins dimers B2, B5, B8, and B2-3" extracted from grape seeds (Vitis vinifera)with a hydroalcoholic solution $(1: 1, v / v)$ and purified by chromatographic techniques as described previously (29). Briefly, the hydroalcoholic extract was fractionated on Toyopearl HW-40 (Tosohaas, Germany) column chromatography using methanol as eluent, and the individual procyanidins were purified by semipreparative HPLC (Merck-Hitachi L-7100) using two connected columns Ultrasphere C18 ODS $(250 \times 4.6 \mathrm{~mm}$ i.d. $)$ according the experimental procedure described elsewhere (29). Final purity was assessed by HPLC-DAD and mass spectrometry.

Before use, all of the compounds were kept under nitrogen atmosphere at $-20{ }^{\circ} \mathrm{C}$. The solutions of procyanidins were freshly prepared prior to the assays.

Plasma Loading with Procyanidins and LDL Isolation. Human plasma from healthy human volunteers was incubated with the procyanidins $(10$ and $50 \mu \mathrm{M})$ at $37^{\circ} \mathrm{C}$ for $1 \mathrm{~h}$, in a closed glass vessel protected from light and under a nitrogen stream. Control plasma, without the compounds, was treated in the same way. LDL was subsequently isolated by density gradient ultracentrifugation as previously described (30). Briefly, plasma was ultracentrifugated in a Beckman L80 ultracentrifuge (Beckman Instruments, Inc., USA) equipped with a fixed angle rotor $70.1 \mathrm{Ti}$, at $65,000 \mathrm{rpm}$, at $15{ }^{\circ} \mathrm{C}$ for $3 \mathrm{~h}$ and the LDL fraction collected with a Pasteur pipet by suction. To minimize LDL oxidation during the isolation process, all of the buffers used were saturated with nitrogen for at least $30 \mathrm{~min}$. Protein concentration was measured by the method of Lowry et al. (31) with bovine serum albumin as standard.

cis-Parinaric Acid Fluorescence Assay. The fluorescence decay of cis-parinaric acid (PnA; Cayman Chemical, USA) incorporated into the LDL outer monolayer has been demonstrated to be a highly sensitive methodology to follow LDL oxidation in its initial steps (32). PnA fluorescence was monitored at $37{ }^{\circ} \mathrm{C}$, in a Perkin-Elmer LS 50 spectrometer (PerkinElmer Life and Analytical Sciences, Inc., USA) provided with a thermostatted cuvette and with a magnetic stirring device. The excitation and the emission wavelengths were 324 and 413 $\mathrm{nm}$, respectively (slit widths $3.5 \mathrm{~nm}$ ). The incorporation of the probe was carried out by adding an aliquot of an ethanolic solution of 1.5 nmol of PnA to $2 \mathrm{~mL}$ of phosphate buffer ( $\mathrm{pH}$ 7.4) containing LDL $(30 \mu \mathrm{g} \mathrm{ApoB})$ and allowed to proceed for about $5 \mathrm{~min}$ with gentle stirring. The oxidation reaction, performed under conditions of controlled radical flux, was initiated by the addition of AAPH solution
(Polysciences, Inc., Germany), to achieve a final concentration of 5 $\mathrm{mM}$, and the fluorescence of PnA recorded. The AAPH solution was freshly prepared in phosphate buffer before use.

Conjugated-Diene Measurements. Conjugated-diene hydroperoxide formation was determined spectrophotometrically in a Perkin-Elmer Lambda 6 UV/vis Spectrometer (PerkinElmer Life and Analytical Sciences, Inc., USA) by continuously monitoring the absorbance at $234 \mathrm{~nm}$. LDL particles ( $30 \mu \mathrm{g}$ of ApoB), isolated from plasma incubated with procyanidins, were added to $2 \mathrm{~mL}$ of phosphate buffer ( $\mathrm{pH} 7.4$ ) containing $5 \mathrm{mM} \mathrm{AAPH}$ in a thermostatted cuvette at $37{ }^{\circ} \mathrm{C}$ with a magnetic stirring device under normal atmosphere and the absorbance monitored for $120 \mathrm{~min}$.

Evaluation of $\alpha$-Tocopherol Consumption. $\alpha$-tocopherol consumption was evaluated in LDL particles isolated from plasma loaded with procyanidins and in LDL directly incubated with the same compounds. In this case, procyanidins $(2 \mu \mathrm{M})$ were added directly to isolated LDL aliquots that had been previously enriched with $4 \mu \mathrm{M} \alpha-\mathrm{TOH}$ for $1 \mathrm{~h}$ at $37^{\circ} \mathrm{C}$, in a closed glass vessel protected from light and under nitrogen stream. Both LDL fractions $(120 \mu \mathrm{g}$ of ApoB) were exposed to $5 \mathrm{mM}$ AAPH at $37^{\circ} \mathrm{C}$, in a closed glass vessel protected from light. Aliquots were removed at 0,10 , and $30 \mathrm{~min}$ and oxidation immediately stopped by the addition of BHT $(0.1 \mathrm{mg} / \mathrm{mL}) . \alpha$-Tocopherol acetate $(4 \mu \mathrm{M})$ was used as an internal standard. $\alpha-\mathrm{TOH}$ concentration in LDL fractions was determined by HPLC as previously described (14). Briefly, $\alpha-\mathrm{TOH}$ was extracted from LDL with hexane, and the organic phase was removed and evaporated under a low pressure nitrogen stream. The resulting sample was reconstituted in $100 \mu \mathrm{L}$ of HPLC mobile phase (95:5 ethanol/isopropanol) and directly injected into the HPLC system (Beckman System Gold, Beckman Instruments, Inc., USA). The separation was performed using a Lichrosphere $100 \mathrm{RP}-18125 \times 4$ $\mathrm{mm}, 5 \mu \mathrm{m}$ particle size reverse phase column, preceded by a Lichrosphere Si $604 \times 45 \mathrm{~mm}$ guard column. The detection of $\alpha$-TOH was carried out at $293 \mathrm{~nm}$.

Quantification of $\alpha$-Tocopheroxyl Radical in Micellar Solutions. A micellar solution of sodium dodecyl sulfate (SDS) was prepared in $50 \mathrm{mM}$ phosphate buffer at $\mathrm{pH}$ 7.4. Micellar dispersions of $\alpha-\mathrm{TOH}$ were prepared by diluting an appropriate volume of an ethanolic solution of $\alpha-\mathrm{TOH}$ into SDS micelles to reach a final concentration of $2 \mathrm{mM}$. The $\alpha-\mathrm{TOH}$-containing micelles were exposed to a UV light source for $3 \mathrm{~min}$ in order to generate $\alpha$-tocopheroxyl radicals $\left(\alpha-\mathrm{TO}^{*}\right)$. Immediately after switching off UV irradiation, procyanidins were added to the micelles at concentrations of $0,5,10,25,50$, and 100 $\mu \mathrm{M}$. Samples were rapidly transferred to bottom-sealed Pasteur pipettes, immediately inserted into the EPR cavity of a Brucker EMX EPR spectrometer, and spectra recorded using the following instrument settings: microwave frequency, $9.8 \mathrm{GHz}$; microwave power, $20 \mathrm{~mW}$; modulation frequency, $100 \mathrm{kHz}$; modulation amplitude, $2 \mathrm{G}$; time constant, $0.65 \mathrm{~s}$. The time interval between switching off UV irradiation and recording of electron paramagnetic resonance spectra was consistently 2 min. Signal areas were converted in $\alpha$-TO ${ }^{*}$ concentrations using a calibration curve constructed with TEMPO (2,2,4,4-tetramethylpiperidine-1oxyl) as standard.

Statistical Analysis. All data are expressed as the mean \pm SEM. Statistical analysis was performed by one way analysis of variance followed by Dunnett's test using the statistical software GraphPad Prism (Version 4.00). A value of $p$ lower than 0.05 was considered statistically significant.

\section{RESULTS}

Oxidation of LDL Isolated from Plasma Incubated with Procyanidins: PnA Fluorescence Decay. The polyunsaturated fatty acid PnA, incorporated into LDL particles, has been successfully used as a fluorescent probe to monitor the initial stages of radical-induced LDL oxidation as well as to evaluate its inhibition by several antioxidants $(32,22)$. LDL fractions isolated from plasma aliquots incubated with the procyanidins, except in the case of B5 dimer, exhibited lower rates of PnA oxidation as compared with the control plasma not supplemented with procyanidins (Figure 2). Table 1 reports the quantification 


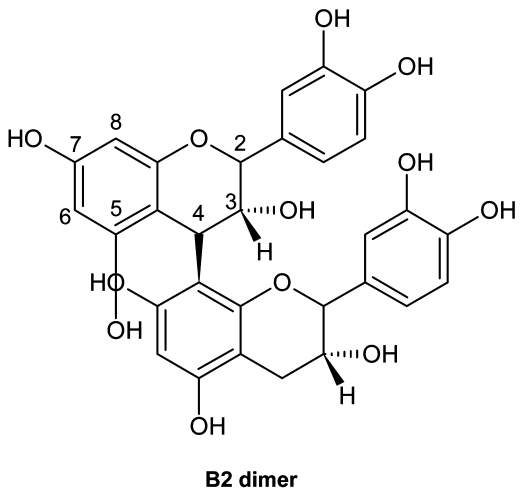

[epicatechin-(4 $\beta$-8)-epicatechin]<smiles>Oc1cc(O)c2c(c1)OC(c1ccc(O)c(O)c1)[C@H](O)[C@@H](O)[C@H]2c1c(O)cc(O)c2c1OC(c1ccc(O)c(O)c1)CC2</smiles>

B2-3"-o-gallate

C1 trimer

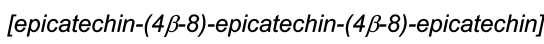<smiles>[R7]C1([R7])[C@@H](c2ccc(O)c(O)c2)Oc2cc(O)cc(O)c2[C@@H]1c1c(O)cc2c(c1O)C[C@@H](O)[C@H](c1ccc(O)c(O)c1)O2</smiles>

B5 dimer $\left(\mathrm{R}_{1}=\mathrm{H}, \mathrm{R}_{2}=\mathrm{OH}\right)$

[epicatechin-(4 $\beta$-6)-epicatechin]

B8 dimer $\left(\mathrm{R}_{1}=\mathrm{OH}, \mathrm{R}_{2}=\mathrm{H}\right)$

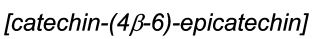

Figure 1. Chemical strutures of red wine procyanidins.

of PnA oxidation in terms of inhibition periods ( $\mathrm{t}$ lag) and of oxidation rate. The LDL isolated from plasma containing B2$3^{\prime \prime}$-o-gallate was the most resistant to oxidation, as inferred from the longest period of inhibition of PnA fluorescence decay and, afterward, the lowest rate of probe oxidation. At a concentration of $10 \mu \mathrm{M}$ in plasma, B2-3"-o-gallate evoked an increase in the inhibition period of PnA oxidation around $42 \%$ and a decrease in its oxidation rate around $10 \%$, in the respective LDL fraction. $\mathrm{PnA}$ is highly sensitive to oxidation, and the apparent small differences observed for the several fractions are significant observations, suggesting that procyanidins are attached to LDL, protecting the particles from AAPH-derived peroxyl radicals and thus reflecting distinct abilities to resist to oxidation.

Moreover, in the case of the dimer B8, but not for the other compounds, a positive correlation between concentration (up to $50 \mu \mathrm{M}$ in plasma) and the protection of PnA oxidation at the LDL surface (data not shown) was found.

Oxidation of LDL Isolated from Plasma Incubated with Procyanidins: Conjugated Dienes Formation. The oxidation of LDL induced by AAPH was also followed by continuously monitoring the formation of relatively stable products of lipid peroxidation, the conjugated dienes hydroperoxides. Figure 3 shows representative time courses of conjugated diene formation in LDL control (nLDL) and in LDL isolated from plasma incubated with $10 \mu \mathrm{M}$ procyanidins (iLDL). In agreement with the PnA results, a reduction in the rate of hydroperoxide formation was observed in LDL isolated from plasma-containing procyanidins as compared with LDL isolated from the same plasma sample but devoid of procyanidins. The C1 trimer, B2$3^{\prime \prime}$-o-gallate and B2 dimer were the most efficient compounds in protecting LDL from oxidation when plasma was incubated with $10 \mu \mathrm{M}$ (Table 2). For the $\mathrm{C} 1$ trimer and B8 dimer, concentration-dependent effects were observed (data not shown).

Oxidation of $\alpha$-Tocopherol in LDL Fractions. In order to bring insights into the mechanism by which LDL isolated from plasma-containing procyanidins better resisted oxidation than LDL isolated from the same plasma but devoid of such compounds (control plasma), the oxidative degradation of $\alpha$-TOH in the LDL fractions challenged with a constant flux of peroxyl radicals was followed. Figure 4A shows the effect of the procyanidins directly added to LDL on $\alpha$-TOH consumption. Under these conditions, procyanidins $(2 \mu \mathrm{M})$ effectively protect $\alpha-\mathrm{TOH}$ degradation as inferred from the rates of $\alpha-\mathrm{TOH}$ 


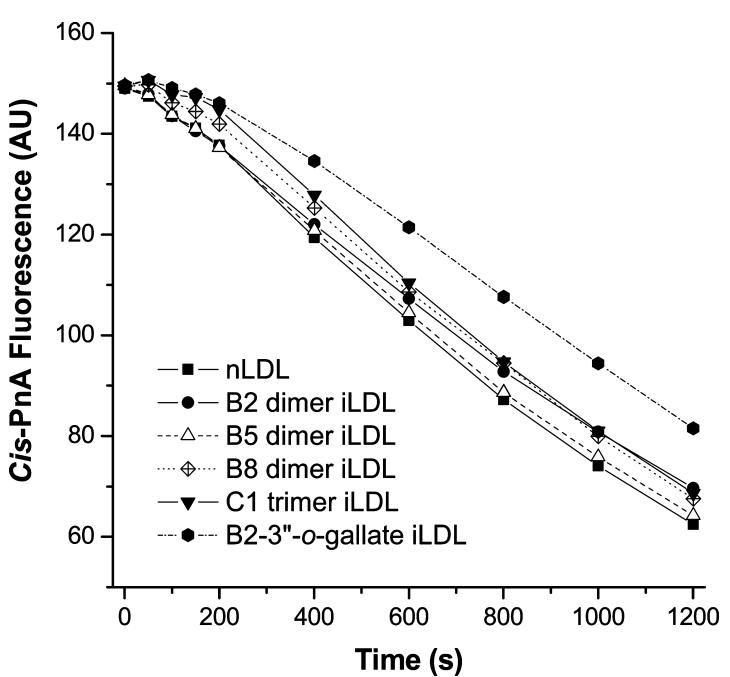

Figure 2. Representative curves of cis-parinaric acid fluorescence decay in AAPH-induced oxidation of LDL control (nLDL) and of LDL particles isolated from plasma incubated with $10 \mu \mathrm{M}$ procyanidins (iLDL): B2 dimer, B5 dimer, B8 dimer, C1 trimer, and B2-3"-o-gallate. The results are representative of three independent experiments.

Table 1. Kinetic Parameters of cis-Parinaric Acid (PnA) Fluorescence Decay in AAPH-Induced Oxidation of LDL Control (nLDL) and LDL Isolated from Plasma Incubated with $10 \mu \mathrm{M}$ Procyanidins (iLDL) ${ }^{a}$

\begin{tabular}{|c|c|c|}
\hline & T lag $[\mathrm{s}]$ & $\begin{array}{c}\text { oxidation rate } \\
\text { [FU/s] }\end{array}$ \\
\hline$n L D L$ & $126.9 \pm 11.9$ & $80.5 \pm 0.7$ \\
\hline B2 dimer iLDL & $134.4 \pm 10.9$ & $74.2 \pm 1.4$ \\
\hline B5 dimer iLDL & $127.6 \pm 11.9$ & $77.8 \pm 0.7$ \\
\hline B8 dimer iLDL & $166.1 \pm 9.5$ & $76.7 \pm 0.7$ \\
\hline C1 trimer iLDL & $178.3 \pm 15.3$ & $80.2 \pm 0.7$ \\
\hline B2-3"-o-gallate iLDL & $181.3 \pm 14.6^{*}$ & $72.4 \pm 3.8^{*}$ \\
\hline
\end{tabular}

${ }^{a}$ The inhibition period of $\mathrm{PnA}$ oxidation (t lag) and the rate of $\mathrm{PnA}$ oxidation (the slope given in fluorescence units per second) are shown. The values represent the mean \pm SEM of three independent experiments. ${ }^{*}$, significantly different from control at $P<0.05$

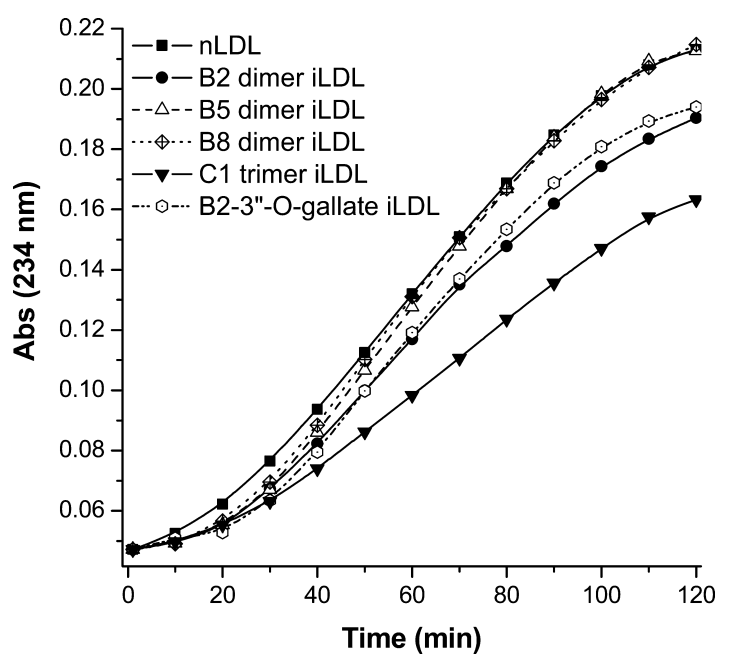

Figure 3. Representative curves of conjugated diene formation in AAPHinduced oxidation in LDL control (nLDL) and in LDL isolated from plasma incubated with $10 \mu \mathrm{M}$ procyanidins (iLDL): B2 dimer, B5 dimer, B8 dimer, $\mathrm{C} 1$ trimer and B2-3"-o-gallate. The results are representative of three independent experiments.

degradation, which are delayed by the phenolic compounds according to the following sequence B2 dimer $>$ B2- $3^{\prime \prime}$ - $O$-gallate $>\mathrm{C} 1$ trimer $>\mathrm{B} 8$ dimer $>\mathrm{B} 5$ dimer. Consistent with this observation, the free radical-induced oxidative degradation of $\alpha$-TOH contained in LDL isolated from plasma preincubated with the phenolic compounds also occurred at rates lower than those in LDL from the plasma control (Figure 4B). Nevertheless, the efficacy observed does not match that found in Figure 4A; LDL isolated from plasma loaded with the B8 dimer were those particles in which $\alpha-\mathrm{TOH}$ was more resistant to depletion, followed by the LDL isolated from plasma loaded with B2-3"$o$-gallate and B2 dimer. Assuming the interaction of the phenolic compounds with the LDL particles, this observation may be explained on the basis of a different partition of the compounds among plasma components.

Reduction of $\alpha$-Tocopheroxyl Radical by the Procyanidins. In 1995, Laranjinha et al. have suggested that polyphenols exhibiting a catechol moiety, similar to vitamin $\mathrm{C}$, are endowed with the reduction capacity to recycle $\alpha$-TOH from its oneelectron oxidation product, the $\alpha$-TO', at LDL and membrane surfaces (14). This has been subsequently verified for distinct compounds, and evidence for such occurrence in vivo has been recently forwarded (33). Considering that such an $\alpha-\mathrm{TOH}$ recycling mechanism, encompassing the transfer of the radical character from lipid compartments to the water phase, affords a high protection of the lipid systems (LDL, membranes) against oxidation (13), we therefore studied the ability of procyanidins to regenerate $\alpha-\mathrm{TOH}$ by reducing $\alpha-\mathrm{TO}^{\circ}$. The $\alpha-\mathrm{TO}^{*}$ was produced by UV irradiation of $\alpha-\mathrm{TOH}$ incorporated into SDS micelles and measured by EPR. The procyanidins and the standard reductants (ascorbate and caffeic acid) were added to the micelles immediately after switching off UV light. Figure 5 shows quantitatively the decay of $\alpha-\mathrm{TO}^{\circ}$ as a function of phenolic concentration, and clearly, all the procyanidins exhibited a concentration-dependent capacity to accelerate the decay of EPR signal intensity, indicating a decrease in the $\alpha$-TO concentration via its reduction to $\alpha$-TOH. B2- $3^{\prime \prime}-o$-gallate was the most efficient compound in suppressing $\alpha-\mathrm{TO}^{\circ}$, exhibiting a potency closer to that of ascorbate, that is, at $5 \mu \mathrm{M}$, it almost completely abolishes the EPR signal. $\mathrm{C} 1$ trimer as well as dimers B2 and B8 also showed a significant ability to remove $\alpha$-TO'. For instance, $10 \mu \mathrm{M}$ of the compounds reduce the radical concentration by c.a. $30-40 \%$. The reduction of $\alpha-\mathrm{TO}^{\circ}$ by the compounds follows the order ascorbate (EC50 $4.2 \mu \mathrm{M})$, B2$3^{\prime \prime}$-o-gallate (EC50 8.9 $\left.\mu \mathrm{M}\right), \mathrm{C} 1$ trimer (EC50 9.5 $\left.\mu \mathrm{M}\right)$, caffeic acid $(16.1 \mu \mathrm{M}), \mathrm{B} 2$ dimer (EC50 21.6 $\mu \mathrm{M}$ ), B8 dimer (EC50 $22.5 \mu \mathrm{M}$ ), and B5 dimer (EC50 52.9 $\mu \mathrm{M})$.

\section{DISCUSSION}

The oxidation of LDL in the intima space of the arterial wall is considered a crucial molecular event in the development of fatty streaks, leading to atherosclerotic lesions $(1,17)$. At such a location, LDL is thought to be out of the protection of plasmatic antioxidants, most notably of ascorbate, a compound capable of maintaining LDL $\alpha-\mathrm{TOH}$ at high steady state concentration under oxidation conditions (17). The notion that phenolic compounds tested as antioxidants in vitro may exert antioxidant effects in plasma faces difficulties based on their limited bioavailability. In fact, although these dietary compounds have been assigned antioxidant activities, largely based on in vitro assays, it is clear that they are extensively biotransformed during absorption and reach the plasma predominantly as glucuronide and sulfate conjugates (7). The unchanged molecules peak at very low concentrations when considering their isotropic dilution in the water phase of blood $(5,34)$. However, because of its physicochemical properties, encompassing an amphyphilic character and the potential to form hydrogen bonds 
Table 2. Kinetic Parameters of Conjugated-Diene Formation in AAPH-Induced Oxidation of LDL Control (nLDL) and LDL Isolated from Plasma Incubated with $10 \mu \mathrm{M}$ Procyanidins (iLDL) ${ }^{a}$

\begin{tabular}{|c|c|c|c|c|}
\hline & $\Delta \mathrm{Abs}$ & $\mathrm{T} 1 / 2$ [min] & $\mathrm{t}$ lag [min] & $\begin{array}{c}\text { oxidation rate } \\
{[\mathrm{OD} / \mathrm{min}] \times 10^{-3}}\end{array}$ \\
\hline $\mathrm{nLDL}$ & $0.166 \pm 0.10$ & $62.2 \pm 1.3$ & $24.7 \pm 1.99$ & $2.1 \pm 0.1$ \\
\hline B8 dimer iLDL & $0.167 \pm 0.1$ & $64.0 \pm 2.1$ & $26.2 \pm 2.4$ & $2.1 \pm 0.1$ \\
\hline $\mathrm{C} 1$ trimer iLDL & $0.122 \pm 0.07^{*}$ & $69.1 \pm 1.4^{*}$ & $29.2 \pm 2.1^{*}$ & $1.5 \pm 0.1^{*}$ \\
\hline B2-3"'-o-gallate iLDL & $0.146 \pm 0.08^{*}$ & $63.1 \pm 0.2$ & $28.7 \pm 1.2^{*}$ & $2.0 \pm 0.1$ \\
\hline
\end{tabular}

${ }^{a}$ The values represent the mean \pm SEM of three independent experiments. ${ }^{*}$, significantly different from control at $P<0.05$.

A)

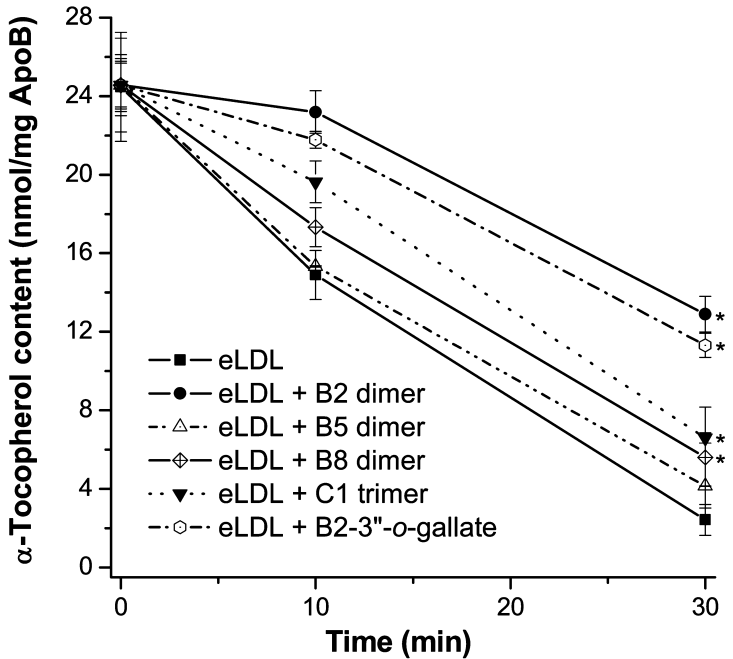

B)

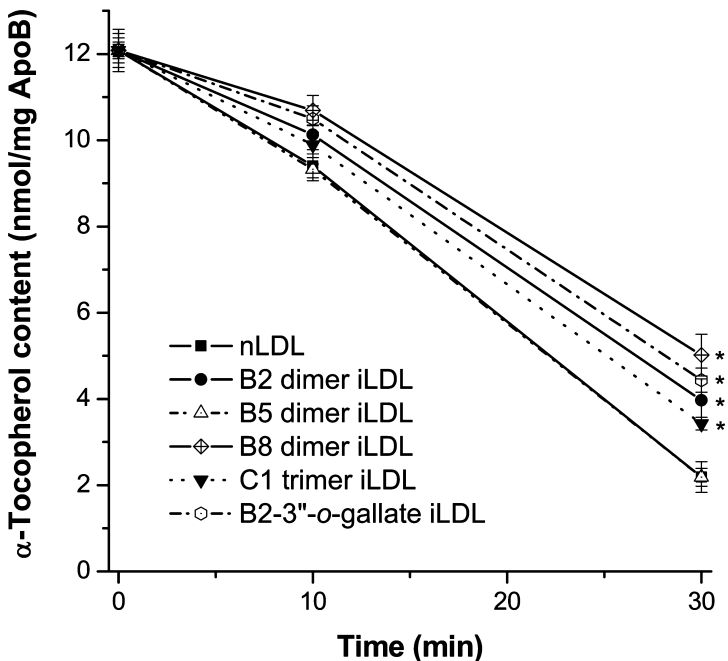

Figure 4. Depletion of $\alpha$-tocopherol over time upon exposure to a constant flux of peroxyl radicals in (A) $\alpha$-tocopherol-enriched LDL (eLDL) incubated with procyanidins $(2 \mu \mathrm{M})$ and (B) LDL (iLDL) isolated from human plasma incubated with procyanidins $(50 \mu \mathrm{M})$. In panel $\mathbf{B}$, symbols representing the values of $n-L D L$ and B5 dimer iLDL are superimposed. Each point represents the mean \pm SEM of three independent experiments. *, significantly different from control at $P<0.05$.

via the several -OH groups, they have been shown to interact with membranes and $\operatorname{LDL}(15,16)$. In fact, following the consumption of phenolic rich food/beverages, a small percentage of unchanged phenolic compounds have been detected in human plasma and, notably, in association with lipoprotein fractions (35). For instance, Aviram et al. (2) have shown that the consumption of red wine, but not of white wine, by healthy volunteers, resulted in the enrichment of their plasma LDL with flavonoids such as quercetin. Thus, it is likely that at membrane surfaces the phenolic compounds may reach a high local

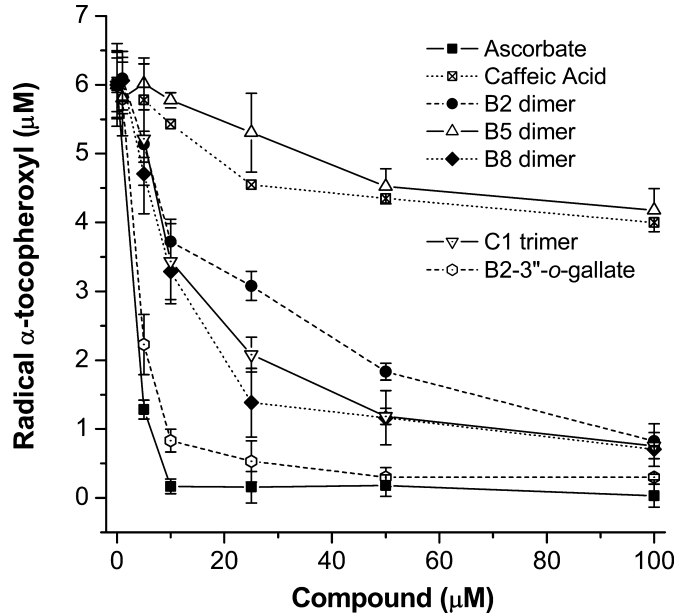

Figure 5. Decay of $\alpha$-tocopheroxyl radicals in micellar solutions of sodium dodecyl sulfate (SDS) incubated with increasing concentrations of procyanidins and with standard reductants. $\alpha-\mathrm{TO}^{\circ}$ was produced by oxidation induced by UV irradiation of SDS micelles containing $\alpha-T O H$. Each point represents the mean \pm SEM of three independent experiments

concentration and be enrolled in redox reactions with $\alpha$-TOH, a mechanism that was shown to be highly efficient in the protection of the lipoprotein particles from oxidation (13). Therefore, the presence of polyphenols at the LDL surface, providing protection against oxidation, would be particular critical in the intima space.

Compatible and in support of this notion, in this work it is demonstrated that LDL isolated from plasma incubated with red wine procyanidins exhibited a significantly higher resistance to oxidation as compared with LDL isolated from the same plasma but devoid of the procyanidins. Technical difficulties prevented us from obtaining clear-cut data for the LDL content in procyanidins. Oxidation was sensitively followed by means of a fluorescent probe incorporated in the LDL outer monolayer (Figure 2) as well as by the formation of conjugated-diene hydroperoxide (Figure 3). The results in part corroborate those obtained before, when the same compounds were directly added to LDL immediately before oxidation (22). The relationship between the flavanol structure of procyanidins and the antioxidant activity against LDL oxidation was then established, in particular that (i) the antioxidant efficiency increases with the degree of polymerization of the compounds, which, for later stages of oxidation, may be related with the number of hydroxyl groups, (ii) the antioxidant efficiency increases by esterification of C3 hydroxyl group with gallic acid, and (iii) the interflavan linkage constitutes an important structural characteristic in the antioxidant efficiency of the compounds (since it affects the molecular conformation and hydrophobicity). Moreover, dimers with the interflavan bond $\mathrm{C} 4-\mathrm{C} 8$ have an increased antioxidant activity in comparison with dimers with the interflavan bond 
C4-C6, which could be related with the more closed conformation adopted by $\mathrm{C} 4-\mathrm{C} 8$ dimers and consequently with the increased hydrophobic character of these molecules (22). In agreement, in this work, the B2 dimer (C4-C8 bond) consistently afforded a significantly higher antioxidant protection in comparison with the B5 and B8 dimers ( $\mathrm{C} 4-\mathrm{C} 8$ bond). Additionally, esterification of the $\mathrm{C} 3$ hydroxyl group with gallic acid also showed an increase in antioxidant activity, but only in the initial stages of LDL oxidation. B2-3" $-o$-gallate showed higher antioxidant activity than the B2 dimer in the PnA assay; however, a significant difference between both compounds in conjugated-diene formation was not observed.

When considering the mechanism of LDL resistance to oxidation, it is likely that although the quenching of AAPHderived peroxyl radicals reaching LDL particles from the water phase by LDL-bound polyphenols may occur, the protection may be, at least in part, assigned to the recycling of $\alpha-\mathrm{TOH}$ at the LDL surface. This conclusion is supported by two interrelated features, namely, (a) the lower rates of $\alpha$-TOH oxidation in LDL obtained from plasma-containing procyanidins when challenged with a constant flux of peroxyl radicals, as compared with LDL isolated from control plasma (Figure 4A) and (b) the ability of the procyanidins to reduce $\alpha-\mathrm{TO}^{\circ}$ as shown by EPR in model micelar systems (Figure 5). Procyanidins $(2 \mu \mathrm{M})$ directly added to $\alpha$-TOH-enriched LDL reduced $\alpha-\mathrm{TOH}$ consumption, 30 min after oxidation induction, between 7 and $47 \%$, with the B2 dimer, and B2-3" $-o$-gallate and C1 trimer being the most efficient compounds. When added to plasma before LDL isolation $(50 \mu \mathrm{M})$, procyanidins were able to reduce $\alpha$-TOH consumption, $30 \mathrm{~min}$ after oxidation induction (until $28 \%$ ) with the $\mathrm{B} 8$ dimer being the most the efficient compound, followed by B2- $3^{\prime \prime}-o$-gallate and the B2 dimer. Actually, the presence of antioxidant compounds capable of regenerating $\alpha-\mathrm{TOH}$ is essential to prevent the described pro-oxidant activity of $\alpha-\mathrm{TOH}$ via the so-called $\alpha$-TOH-mediated peroxidation, resulting in the oxidation of polyunsaturated lipids in LDL particles (36). This implies that $\alpha$-TOH acts as an effective antioxidant only in coexistence with ascorbate or with other compounds able to eliminate $\alpha$-TO ${ }^{\bullet}$ produced in the peroxidation process and to regenerate $\alpha-\mathrm{TOH}$, transferring the radical character to the water phase. Such a property to reduce $\alpha$-TO was already described for related compounds, namely, caffeic acid and some green tea polyphenols $(14,37)$. The prevention of $\alpha$-TOH oxidation in LDL by red wine phenolics has also been demonstrated to preserve endothelium function via the inhibition of lipid oxidation (38). Finally, the analysis of the results requires the consideration of the concentration of the phenols added to plasma. It may be realized that the high concentration of the phenols added to the plasma, as compared with in vivo concentrations, were necessary to demonstrate in vitro the proof of principle for the potential protective effects at the LDL level under chronic low level oxidation events.

In conclusion, the present study shows that LDL isolated from plasma-containing red wine procyanidins is endowed with an increased resistance to oxidation in terms of lipid peroxidation that may be, at least in part, ascribed to the $\alpha$-TOH-recycling ability of the phenolic compounds associated with the lipoprotein particles. Of note, this effect is influenced by the structural features of procyanidins. Therefore, the notion based on the location of phenolic compounds, bound to LDL and the local antioxidant effects, exporting the radical character from the lipoprotein-lipid phase to the water phase, may help reconcile apparently colliding observations relating the health benefits of dietary phenolic antioxidants and the low concentrations achieved in plasma for the unchanged molecules, when considering their isotropic dilution in human plasma.

\section{ABBREVIATIONS USED}

AAPH, 2,2'-azo-bis(2-amidino-propane hydrochloride); BHT, butylated hydroxytoluene; EPR, electron paramagnetic resonance; LDL, low density lipoprotein; PnA, cis-parinaric acid; $\alpha$-TO, $\alpha$-tocopheroxyl radical; $\alpha$-TOH, $\alpha$-tocopherol.

\section{LITERATURE CITED}

(1) Witztum, J. L.; Steinberg, D. The oxidative modification hypothesis of atherosclerosis: does it hold for humans. Trends Cardiovasc. Med. 2001, 11, 93-102.

(2) Aviram, M.; Fuhrman, B. Wine flavonoids protect against LDL oxidation and atherosclerosis. Ann. N.Y. Acad. Sci. 2002, 957, 146-61.

(3) Berliner, J. A.; Heinecke, J. W. The role of oxidized lipoproteins in atherogenesis. Free Radical Biol. Med. 1996, 20, 707-27.

(4) Bors, W.; Heller, W.; Michel, C.; Saran, M. Flavonoids as antioxidants: determination of radical-scavenging efficiencies. Methods Enzymol. 1990, 186, 343-55.

(5) Simonetti, P.; Gardana, C.; Pietta, P. Caffeic acid as biomarker of red wine intake. Methods Enzymol. 2001, 335, 122-30.

(6) Walle, T. Absorption and metabolism of flavonoids. Free Radical Biol. Med. 2004, 36, 829-37.

(7) Rice-Evans, C. Flavonoids and isoflavones: absorption, metabolism, and bioactivity. Free Radical Biol. Med. 2004, 36, 827-8.

(8) De Azevedo, W. F., Jr.; Mueller-Dieckmann, H. J.; SchulzeGahmen, U.; Worland, P. J.; Sausville, E.; Kim, S. H. Structural basis for specificity and potency of a flavonoid inhibitor of human CDK2, a cell cycle kinase. Proc. Natl. Acad. Sci. U.S.A. 1996, 93, 2735-40.

(9) Williams, R. J.; Spencer, J. P.; Rice-Evans, C. Flavonoids: antioxidants or signalling molecules. Free Radical Biol. Med. 2004, 36, 838-49.

(10) Corder, R.; Mullen, W.; Khan, N. Q.; Marks, S. C.; Wood, E. G.; Carrier, M. J.; Crozier, A. Oenology: red wine procyanidins and vascular health. Nature 2006, 444, 566.

(11) Rahman, I.; Biswas, S. K.; Kirkham, P. A. Regulation of inflammation and redox signaling by dietary polyphenols. Biochem. Pharmacol. 2006, 72, 1439-52.

(12) Steffen, Y.; Schewe, T.; Sies, H. (-)-Epicatechin elevates nitric oxide in endothelial cells via inhibition of NADPH oxidase. Biochem. Biophys. Res. Commun. 2007, 359, 828-33.

(13) Laranjinha, J.; Cadenas, E. Redox cycles of caffeic acid, alphatocopherol, and ascorbate: implications for protection of lowdensity lipoproteins against oxidation. IUBMB Life 1999, 48, 5765 .

(14) Laranjinha, J.; Vieira, O.; Madeira, V.; Almeida, L. Two related phenolic antioxidants with opposite effects on vitamin E content in low density lipoproteins oxidized by ferrylmyoglobin: consumption vs regeneration. Arch. Biochem. Biophys. 1995, 323, 373-81.

(15) Terao, J.; Piskula, M. K. Flavonoids and membrane lipid peroxidation inhibition. Nutrition 1999, 15, 790-1.

(16) Fraga, C. G. Plant polyphenols: how to translate their in vitro antioxidant actions to in vivo conditions. IUBMB Life 2007, 59, 308-15.

(17) Stocker, R.; Keaney, J. F., Jr. Role of oxidative modifications in atherosclerosis. Physiol Rev 2004, 84, 1381-478.

(18) Waterhouse, A. L. Wine phenolics. Ann. N.Y. Acad. Sci. 2002, 957, 21-36.

(19) Nigdikar, S. V.; Williams, N. R.; Griffin, B. A.; Howard, A. N. Consumption of red wine polyphenols reduces the susceptibility of low-density lipoproteins to oxidation in vivo. Am. J. Clin. Nutr. 1998, 68, 258-65.

(20) Fuhrman, B.; Aviram, M. Flavonoids protect LDL from oxidation and attenuate atherosclerosis. Curr. Opin. Lipidol. 2001, 12, 418 . 
(21) Howard, A.; Chopra, M.; Thurnham, D.; Strain, J.; Fuhrman, B.; Aviram, M. Red wine consumption and inhibition of LDL oxidation: what are the important components. Med. Hypotheses 2002, 59, 101-4.

(22) da Silva Porto, P. A.; Laranjinha, J. A.; de Freitas, V. A. Antioxidant protection of low density lipoprotein by procyanidins: structure/activity relationships. Biochem. Pharmacol. 2003, 66, 947-54.

(23) Pace-Asciak, C. R.; Hahn, S.; Diamandis, E. P.; Soleas, G.; Goldberg, D. M. The red wine phenolics trans-resveratrol and quercetin block human platelet aggregation and eicosanoid synthesis: implications for protection against coronary heart disease. Clin. Chim. Acta 1995, 235, 207-19.

(24) Pace-Asciak, C. R.; Rounova, O.; Hahn, S. E.; Diamandis, E. P.; Goldberg, D. M. Wines and grape juices as modulators of platelet aggregation in healthy human subjects. Clin. Chim. Acta 1996, 246, 163-82.

(25) Halpern, M. J.; Dahlgren, A. L.; Laakso, I.; Seppanen-Laakso, T.; Dahlgren, J.; McAnulty, P. A. Red-wine polyphenols and inhibition of platelet aggregation: possible mechanisms, and potential use in health promotion and disease prevention. J. Int. Med. Res. 1998, 26, 171-80.

(26) Dell'Agli, M.; Busciala, A.; Bosisio, E. Vascular effects of wine polyphenols. Cardiovasc. Res. 2004, 63, 593-602.

(27) Fumagalli, F.; Rossoni, M.; Iriti, M.; di Gennaro, A.; Faoro, F.; Borroni, E.; Borgo, M.; Scienza, A.; Sala, A.; Folco, G. From field to health: a simple way to increase the nutraceutical content of grape as shown by NO-dependent vascular relaxation. J. Agric. Food Chem. 2006, 54, 5344-9.

(28) Ivanov, V.; Carr, A. C.; Frei, B. Red wine antioxidants bind to human lipoproteins and protect them from metal ion-dependent and -independent oxidation. J. Agric. Food Chem. 2001, 49, 44429.

(29) Freitas, V. D.; Glories, Y.; Bourgeois, G.; Vitry, C. Characterisation of oligomeric and polymeric procyanidins from grape seeds by Liquid Secondary Ion Mass Spectrometry (LSIMS). Phytochemistry 1998, 49, 1434-1441.

(30) Vieira, O. V.; Laranjinha, J. A.; Madeira, V. M.; Almeida, L. M. Rapid isolation of low density lipoproteins in a concentrated fraction free from water-soluble plasma antioxidants. J. Lipid Res. 1996, 37, 2715-21.
(31) Lowry, O. H.; Rosebrough, N. J.; Farr, A. L.; Randall, R. J. Protein measurement with the Folin phenol reagent. J. Biol. Chem. 1951, 193, 265-75.

(32) Laranjinha, J. A.; Almeida, L. M.; Madeira, V. M. Lipid peroxidation and its inhibition in low density lipoproteins: quenching of cis-parinaric acid fluorescence. Arch. Biochem. Biophys. 1992, 297, 147-54.

(33) Frank, J.; Budek, A.; Lundh, T.; Parker, R. S.; Swanson, J. E.; Lourenco, C. F.; Gago, B.; Laranjinha, J.; Vessby, B.; KamalEldin, A. Dietary flavonoids with a catechol structure increase alpha-tocopherol in rats and protect the vitamin from oxidation in vitro. J. Lipid Res. 2006, 47, 2718-25.

(34) Holt, R. R.; Lazarus, S. A.; Sullards, M. C.; Zhu, Q. Y.; Schramm, D. D.; Hammerstone, J. F.; Fraga, C. G.; Schmitz, H. H.; Keen, C. L. Procyanidin dimer B2 [epicatechin-(4beta-8)-epicatechin] in human plasma after the consumption of a flavanol-rich cocoa. Am. J. Clin. Nutr. 2002, 76, 798-804.

(35) Bonanome, A.; Pagnan, A.; Caruso, D.; Toia, A.; Xamin, A.; Fedeli, E.; Berra, B.; Zamburlini, A.; Ursini, F.; Galli, G. Evidence of postprandial absorption of olive oil phenols in humans. Nutr. Metab. Cardiovasc. Dis. 2000, 10, 111-20.

(36) Bowry, V. W.; Ingold, K. U.; Stocker, R. Vitamin E in human low-density lipoprotein. When and how this antioxidant becomes a pro-oxidant. Biochem. J. 1992, 288 (Pt 2) , 341-4.

(37) Zhou, B.; Wu, L. M.; Yang, L.; Liu, Z. L. Evidence for alphatocopherol regeneration reaction of green tea polyphenols in SDS micelles. Free Radical Biol. Med. 2005, 38, 78-84.

(38) Deckert, V.; Desrumaux, C.; Athias, A.; Duverneuil, L.; Palleau, V.; Gambert, P.; Masson, D.; Lagrost, L. Prevention of LDL alphatocopherol consumption, cholesterol oxidation, and vascular endothelium dysfunction by polyphenolic compounds from red wine. Atherosclerosis 2002, 165, 41-50.

Received for review November 14, 2007. Revised manuscript received January 30, 2008. Accepted February 12, 2008. This work was supported by a grant from Fundação para a Ciência e Tecnologia (project grant POCTI/AGR/42418/2001and PTDC/AGR-ALI/71262/ 2006). C.L. was supported by the fellowship SFRH/BD/27333/2006.

JF0733259 